\title{
Lithium-Ion Conductivity in Lithium Lanthanum Titanates as Different Local Distortion Model Compounds
}

\author{
Toyoki Okumura, ${ }^{\mathrm{a}, *}$ Tomokazu FukUtsuka, ${ }^{\mathrm{a}}$ Yoshiharu Uснімото, \\ Morihiro SAITO, ${ }^{b}$ and Jun KUWANO ${ }^{b}$
}

\begin{abstract}
${ }^{\mathrm{a} G r a d u a t e}$ School of Human and Environmental Studies, Kyoto University (Yoshida-nihonmatsu-cho, Sakyo-ku, Kyoto 606-8501, Japan)

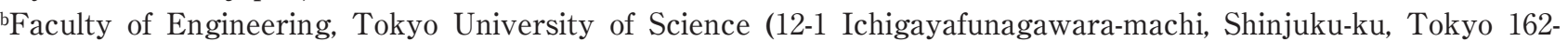
0826, Japan)
\end{abstract}

Received December 14, 2009 ; Accepted February 23, 2010

\begin{abstract}
The extended X-ray absorption fine structure (EXAFS) was measured to study the effects of the local structures on the $\mathrm{Li}$-ion transfer in the $\mathrm{La}_{2 / 3-\mathrm{x}} \mathrm{Li}_{3 \mathrm{x}} \mathrm{TiO}_{3}$ perovskite model compound with different lanthanum ion concentrations. The results indicate that the local structure is more distorted and the interatomic distances of Ti-La and TiO-Ti become shorter with the decrease of lanthanum ion concentration. The change in Li-ion conductivity with different lanthanum ion concentration is well explained by the relation between the estimation of activation energy with which Li ions hop through the bottleneck and the results of local distortion by EXAFS analysis.
\end{abstract}

Key Words : $\mathrm{La}_{2 / 3-\mathrm{x}} \mathrm{Li}_{3 \mathrm{x}} \mathrm{TiO}_{3}$, EXAFS, Li-ion Batteries

\section{Introduction}

The increase of the lithium-ion conductivity in insertion electrodes and/or solid-state electrolyte materials is one of the crucial terms to develop high power lithium batteries, which include all-solid-state lithium-ion battery. The fundamental study of the relationship between structural change and lithium-ion transfer indicates the way for the improvement of lithium-ion conductivity in inorganic materials.

Much attention has been paid to $\mathrm{La}_{2 / 3-\mathrm{x}} \mathrm{Li}_{3 \mathrm{x}} \mathrm{TiO}_{3}$ (LLT) perovskite oxides with A-site deficiency for the development of solid-state electrolyte because of their high lithium-ion conductivities, $c a \cdot 10^{-3}-10^{-5} \mathrm{~S} \mathrm{~cm}^{-1}$ at room temperature. Thus, many researchers have already studied the clarification of their high lithium-ion conductivity. From these results, the reasons for LLT having high lithium-ion conductivity described as follow: 1) lithium ion, lanthanum ion and vacancy are distributed to the A site of LLT perovskite structure, 2) large lanthanum ions maintain the framework of LLT perovskite structure, 3) lithium ion is relatively easy to hop through the bottleneck consisted of four $\mathrm{TiO}_{6}$ octahedra compared with the other compounds. LLT is a model compound for the research of the relationship between the local structural change with the distribution of ions and their lithium-ion transfer since the concentrations of each ion in $\mathrm{La}_{2 / 3-\mathrm{x}} \mathrm{Li}_{3 \mathrm{x}} \mathrm{TiO}_{3}$ can be easily controlled from $\mathrm{x}=0.09$ to 0.15 .

Average structures of LLT were investigated by diffraction techniques. ${ }^{1-11)}$ In the earlier reports, it has been recognized that relatively weak superlattice reflections in X-ray diffraction (XRD) pattern implied tetragonal phases with $a_{p} \times a_{p} \times 2 a_{p}$ ( $p$ refers to the cubic perovskite-type structure), and corresponded to the alterna- tive arrangements of the La-rich layers and the La-poor (Li-vacancy-rich) layers along the c-axis. ${ }^{10)}$ The order of A-site cations in LLT depends on the sintering condition; annealing from higher temperature and quenching weaken the superlattice reflections. ${ }^{11)}$ Furthermore, Harada $e t$ $a l$. reported that the degree of the alternative arrangements of the A-site cations affects the Li ion conduction in LLT. The $\alpha$-LLT (space group: Pm $3 m, X=0.12$ ) with disordered arrangement of the A-site cations showed the highest ionic conductivity among all the polymorphs of perovskite compounds, and the $\beta$-LLT (space group: $P 4 / \mathrm{mmm}$ ) with the ordered arrangements of the A-site cations along the c-axis showed lower conductivity. ${ }^{12,13)}$ Recently, orthorhombic large superstructures (e.g. $2 \mathrm{a}_{\mathrm{p}} \times$ $2 \mathrm{a}_{\mathrm{p}} \times 2 \mathrm{a}_{\mathrm{p}}^{1,6,7)}$ or $\left.\sqrt{2} \mathrm{a}_{\mathrm{p}} \times \sqrt{2} \mathrm{a}_{\mathrm{p}} \times 2 \mathrm{a}_{\mathrm{p}}^{5,14)}\right)$ with the anti-phase tilting of $\mathrm{TiO}_{6}$ octahedral in LLT with various concentrations of $\mathrm{Li}$ ions and sintering conditions were proposed with Rietveld structural refinement with electron or neutron diffraction analysis. However, numerous reports about the complex structures of LLT were only with diffraction techniques.

The extended X-ray absorption fine structure (EXAFS) technique, which provided with the local environment around particular atoms, has been a powerful tool to understand the coefficient between the conductivities and the local structures of materials, ${ }^{15-17)}$ which is difficult to measure with the diffraction techniques. In this paper, we research the relationship between the local structure around titanium ion and their lithium-ion transfer in quenched $\mathrm{La}_{2 / 3-\mathrm{x}} \mathrm{Li}_{3 \mathrm{x}} \mathrm{TiO}_{3}(\mathrm{x}=0.09,0.11,0.15)$.

\section{Experimental}

Quenched samples of $\mathrm{La}_{2 / 3-\mathrm{x}} \mathrm{Li}_{3 \mathrm{x}} \mathrm{TiO}_{3}(\mathrm{x}=0.09,0.11$, $0.15)$ were prepared by a conventional solid state reac- 
Table 1 The list of the lattice parameters (Space group: Cmmm, $\left.a\left(\approx 2 a_{p}\right) \times b\left(\approx 2 a_{p}\right) \times c\left(\approx 2 a_{p}\right)\right)$ obtained by XRD measurement and the conductivity at $25{ }^{\circ} \mathrm{C}$ and the activation energy measured by AC impedance analyzer for $\mathrm{La}_{2 / 3-\mathrm{x}} \mathrm{Li}_{3 \mathrm{x}} \mathrm{TiO}_{3}(\mathrm{x}=0.09,0.11,0.15)$.

\begin{tabular}{|c|c|c|c|c|c|}
\hline \multirow[b]{3}{*}{ Samples } & \multicolumn{3}{|c|}{ XRD data } & \multicolumn{2}{|c|}{ Impedance analysis } \\
\hline & \multicolumn{3}{|c|}{ Lattice parameter } & \multicolumn{2}{|c|}{ Conductivity $\left(25^{\circ} \mathrm{C}\right)$ Activation energy } \\
\hline & $a / \AA$ & $b / \AA$ & $c / \AA$ & $\sigma / \mathrm{Scm}^{-1}$ & $E a / \mathrm{eV}$ \\
\hline $\mathrm{La}_{0.58} \mathrm{Li}_{0.27} \mathrm{TiO}_{3}$ & 7.742 & 7.739 & 7.745 & $8.54 \times 10^{-4}$ & 0.325 \\
\hline $\mathrm{La}_{0.56} \mathrm{Li}_{0.33} \mathrm{TiO}_{3}$ & 7.739 & 7.736 & 7.742 & $1.42 \times 10^{-3}$ & 0.329 \\
\hline $\mathrm{La}_{0.52} \mathrm{Li}_{0.45} \mathrm{TiO}_{3}$ & 7.729 & 7.725 & 7.734 & $6.01 \times 10^{-4}$ & 0.331 \\
\hline
\end{tabular}

tion. Reagent grade $\mathrm{La}_{2} \mathrm{O}_{3}, \mathrm{LiCO}_{3}$ and $\mathrm{TiO}_{2}$ were heated initially at $650^{\circ} \mathrm{C}$ for $2 \mathrm{~h}$ to expel $\mathrm{CO}_{2}$ gas and calcined at $800{ }^{\circ} \mathrm{C}$ for $12 \mathrm{~h}$ on $\mathrm{Au}$ boat. The reground products were cold-pressed into pellets, which were sintered at $1350^{\circ} \mathrm{C}$ for $2 \mathrm{~h}$ on $\mathrm{Pt}$ boats. The samples were prepared by quenching the pellets from the sintering temperature to liquid $\mathrm{N}_{2}$ temperature.

Powder X-ray diffraction (XRD) patterns were measured at room temperature to scan the phases of all powder samples with a Rigaku RINT-UltimaIII system $(\mathrm{Cu}-\mathrm{K} \alpha$ radiation, curved graphite monochromator). Rietveld crystal structure refinements were performed using a RIETAN-2000 program with measured XRD patterns for measuring the lattice parameter of each sample. ${ }^{18)} \mathrm{A}$ conductivity cell, whose opposite faces were polished and spattered gold electrodes, was placed on a measurement jig at Ar atmosphere in steinless chambers. AC impedance measurements were performed with an AC impedance analyzer (YHP 4192A) over a frequency range, 100 $\mathrm{Hz}-1 \mathrm{MHz}$. The conductivities at $25^{\circ} \mathrm{C}$ and the activation energy in a range of $-50-50^{\circ} \mathrm{C}$ estimated from circular arc of Nyquist plots represented the bulk conductivity.

Ti $K$-edge X-ray absorption fine structures of samples were measured by transmission mode using synchrotron radiation at BL-7C, Photon Factory, Tsukuba, Japan. Energy selection was accomplished with a monochromator with $\mathrm{Si}(111)$ crystals, and the intensity of X-ray before-and-after sample was measured using ion chambers. Theoretical parameters used in the curve-fitting analysis were calculated by FEFF8. ${ }^{19,20)}$

\section{Results and Discussion}

The estimated lattice parameters, bulk conductivities at $25^{\circ} \mathrm{C}$ and activation energy in quenched-LLT $(\mathrm{x}=0.09$, $0.11,0.15)$ are listed in Table 1 . The reflection peaks of all the samples were indexed in the perovskite structure [space group: $C m m m, a\left(=2 \mathrm{a}_{\mathrm{p}}\right) \times b\left(=2 \mathrm{a}_{\mathrm{p}}\right) \times c\left(\times 2 \mathrm{a}_{\mathrm{p}}\right)$ ]. The lattice and refined crystal parameters of quenched-LLT $(x=0.09,0.11,0.15)$ were estimated from Rietveld refinements of the crystal structures. The lattice parameters at each axis in all the samples were similar and the crystal structures were pseudo-cubic phase. This is because the $\mathrm{La}^{3+}$ ions, $\mathrm{Li}^{+}$ions, and vacancies were randomly distributed over the A-sites, and the long-range lattice distances at each axis were average values. The lattice parameter of LLT decreased with the decrease of lanthanum-ion concentrations. The maximum conductivity

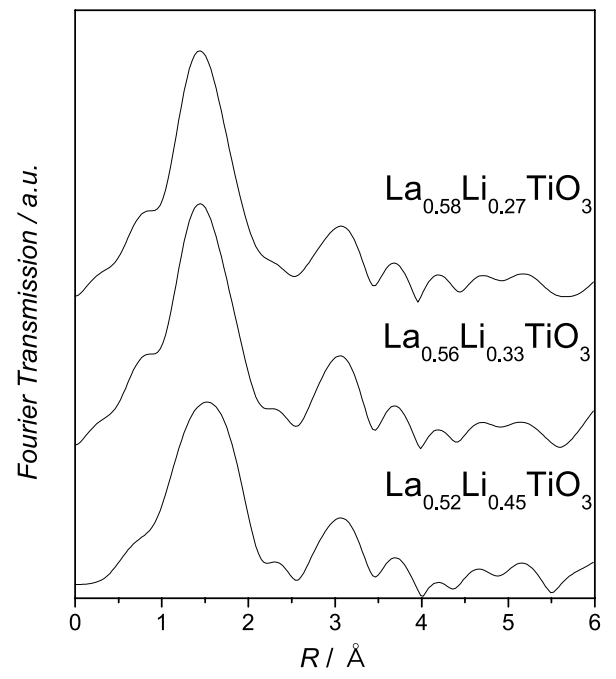

Fig. 1 The pseudo-radial structure functions for $\mathrm{La}_{2 / 3-\mathrm{x}}$ $\mathrm{Li}_{3 \mathrm{x}} \mathrm{TiO}_{3}(\mathrm{x}=0.09,0.11,0.15)$ around $\mathrm{Ti}$ ion obtained by Ti $K$ edge EXAFS spectra.

was $1.42 \times 10^{-3} \mathrm{~S} \mathrm{~cm}^{-1}$ in quenched-LLT at $\mathrm{x}=0.11$. The conductivities of quenched-LLT increased until $\mathrm{x}=0.11$ and decreased at $\mathrm{x}=0.15$. The curve of the conductivity changes formed an arch, since the preferred condition for the $\mathrm{Li}^{+}$ion conduction in LLT is optimized by the $\mathrm{Li}^{+}$ ion and vacancy concentrations, as was previously reported. ${ }^{10)}$ To explore the detailed relationships between the conductivity and structural factors, activation energies $\left(E_{a}\right)$ of quenched-LLT were estimated. The activation energies increased with $\mathrm{x}$.

Figure 1 shows the Fourier transformed (FT) EXAFS of the Ti $K$-edge for quenched-LLT. The FTs of the $k^{3}$ weighted Ti $K$-edge EXAFS oscillations for the samples were calculated within $k=2.80-10.70 \AA^{-1}$ (range was chosen to minimize noise). The pseudo-radial structure functions of the Ti $K$-edge EXAFS show that the first peak at $\sim 1.4 \AA$ corresponded to Ti-O interactions, and that the peaks at $\sim 3.2 \AA$ and $\sim 3.7 \AA$ corresponded to the sum of the Ti-La interactions and the Ti-O-Ti interactions. It was difficult to determine the Ti-Li interactions, since the displacements of the Li ions were expected in a few different positions due to the high mobility of lithium. However, in EXAFS, the Ti-Li interaction was negligible, because the back-scattering ability of the Li atom is very low. The RSFs around the Ti atoms were inversely Fourier-filtered over the first three peaks $(\mathrm{R}=$ 
Table 2 The list of Ti $K$-edge EXAFS determined structural parameter, coordination number (CN), interatomic distance $(R)$, Debye-Waller factors $(\sigma)$, for $\mathrm{La}_{2 / 3-\mathrm{x}} \mathrm{Li}_{3 \mathrm{x}} \mathrm{TiO}_{3}(\mathrm{x}=0.09,0.11,0.15)$.

\begin{tabular}{|c|c|c|c|c|c|c|c|c|c|c|}
\hline & \multicolumn{3}{|c|}{ Ti-O } & \multicolumn{3}{|c|}{ Ti-La } & \multicolumn{3}{|c|}{ Ti-O-Ti } & \multirow{2}{*}{ Residue(\%) } \\
\hline & $\mathrm{CN}$ & $R / \AA$ & $\sigma / \AA$ & $\mathrm{CN}$ & $R / \AA$ & $\sigma / \AA$ & $\mathrm{CN}$ & $R / \AA$ & $\sigma / \AA$ & \\
\hline $\mathrm{La}_{0.58} \mathrm{Li}_{0.27} \mathrm{TiO}_{3}$ & 6 & $1.957(7)$ & $0.095(12)$ & 4.64 & $3.413(17)$ & $0.080(23)$ & 6 & $3.893(32)$ & $0.116(40)$ & 6.013 \\
\hline $\mathrm{La}_{0.56} \mathrm{Li}_{0.33} \mathrm{TiO}_{3}$ & 6 & $1.958(7)$ & $0.096(12)$ & 4.48 & $3.412(15)$ & $0.072(22)$ & 6 & $3.882(29)$ & $0.113(39)$ & 5.846 \\
\hline $\mathrm{La}_{0.52} \mathrm{Li}_{0.45} \mathrm{TiO}_{3}$ & 6 & $1.958(7)$ & $0.105(11)$ & 4.16 & $3.391(14)$ & $0.090(20)$ & 6 & $3.849(33)$ & $0.145(41)$ & 7.782 \\
\hline
\end{tabular}

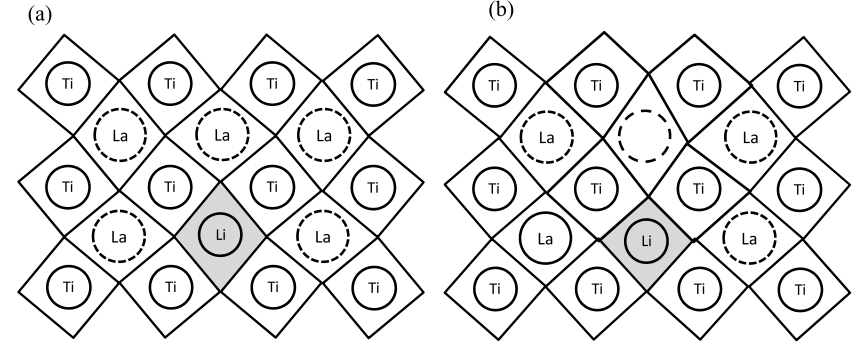

Fig. 2 Schematic figures for the models of local structure for $\mathrm{La}_{2 / 3-\mathrm{x}} \mathrm{Li}_{3 \mathrm{x}} \mathrm{TiO}_{3}$ in which (a) lithium ion is surrounded by lanthanum ion and (b) A-site vacancy consists neighborhood of lithium ion, respectively.

$0.77-4.02 \AA$ ) in the transformation and fitted. The error bars of the atomic distances and DW factors, especially for the Ti-O-Ti interactions, had relatively large values because the selected lengths of the Ti K-edge EXAFS oscillations were short. Therefore, we focused on the relationship between the $\mathrm{x}$ value changes rather than on quantitative values.

Each structural parameter was determined using curve-fitting, and the atomic distances and DW factors were compared to the $\mathrm{x}$ values (Table 2). The local structure was more distorted and the interatomic distances of Ti-La and Ti-O-Ti became shorter with the decrease of lanthanum ion concentration.

Figure 2 shows the simplified LLT models with different A-site vacancy concentrations around the bottleneck consisted of four $\mathrm{TiO}_{6}$ octahedra. The bottleneck, which is the conduction path for lithium ion, was distorted with the increase of $\mathrm{x}$, because the crystal structure was distorted due to the decrease of the number of $\mathrm{La}^{3+}$ ions, which stabilize the perovskite framework and increase the mismatch between the lattices. These facts were observed by the Ti K-edge EXAFS, in which the atomic distances between Ti-La and Ti-O-Ti were decreased. These results were further enhanced with the decrease of the LLT lattice parameter with increasing $\mathrm{x}$ values. Thus, the activation energy increased with increasing $\mathrm{x}$ values.

In conclusion, the differences in the lithium-ion transfer with changes in lanthanum ion concentration were well explained by interactions between lithium and the other ions. The analysis revealed the local distortion around the $\mathrm{Ti}^{4+}$ ion with increases in $\mathrm{x}$, reflecting decreases in the $\mathrm{La}^{3+}$ ion concentration and in the Ti-La and Ti-O-Ti atomic distances. These results indicate that the increase of activation energy of the bulk lithium-ion conduction was caused by the large repulsion force between the $\mathrm{Li}$ ion and the oxygen around the more distorted bottleneck.

\section{References}

1) H. T. Chung and D. S. Cheong, Solid State Ionics, 120, 197 (1999).

2) J. A. Alonso, J. Sanz, J. Santamaría, C. León, A. Várez, and M. T. Fernández-Díaz, Angew. Chem., Int. Ed., 39, 619 (2000).

3) A. Várez, Y. Inaguma, M. T. Fernández-Díaz, J. A. Alonso, and J. Sanz, Chem. Mater., 15, 4637 (2003).

4) J. Sanz, A. Várez, J. A. Alonso, and M. T. FernándezDíaz, J. Chem. Soc., 177, 1157 (2004).

5) S. García-Martín, M. A. Alario-Franco, H. Ehrenberg, J. Rodríguez-Carvajal, and U. Amador, J. Am. Chem. Soc., 126, 3587 (2004).

6) M. Yashima, M. Itoh, Y. Inaguma, and Y. Morii, J. Am. Chem. Soc., 127, 3491 (2005).

7) Y. Inaguma, T. Katsumata, M. Itoh, Y. Morii, and T. Tsurui, Solid State Ionics, 177, 3037 (2006).

8) M. Sommariva and M. Catti, Chem. Mater., 18, 2411 (2006).

9) A. G. Belous, G. N. Novitskaya, S. V. Polyanetskaya, and Y. I. Gornikov, Russ. J. Inorg. Chem., 32, 156 (1987).

10) Y. Inaguma, C. Liquan, M. Itoh, T. Nakayama, T. Uchida, H. Ikuto, and M. Wakihara, Solid State Commun., 86, 689 (1993).

11) H. Kawai and J. Kuwano, J. Electrochem. Soc., 141, 278 (1994).

12) Y. Harada, T. Ishigaki, H. Kawai, and J. Kuwano, Solid State Ionics, 108, 407 (1998).

13) Y. Harada, Y. Hirakoso, H. Kawai, and J. Kuwano, Solid State Ionics, 121, 245 (1999).

14) A. Várez, F. Garcia-Alvarado, A. D. Rovertson, A. R. West, and M. A. Alario-Franco, J. Solid State Chem., 118, 78 (1995).

15) M. Nakayama, H. Ikuta, Y. Uchimoto, and M. Wakihara, Appl. Phys. Lett., 81, 2977 (2002).

16) M. Nakayama, H. Ikuta, Y. Uchimoto, and M. Wakihara, Appl. Phys. Lett., 84, 4227 (2004).

17) M. Nakayama, T. Usui, Y. Uchimoto, M. Wakihara, and M. Yamamoto, J. Phys. Chem. B, 109, 4135 (2005).

18) F. Izumi and T. Ikeda, Mater. Sci. Forum, 321-324, 198 (2000).

19) Rigaku EXAFS Analysis Software, REX2000, Cat. No. 532337031AD, Rigaku Co., 2000.

20) A. L. Ankudinov, B. Ravel, J. J. Rehr, and S. D. Conradson, Phys. Rev. B, 58, 7565 (1998); A. L. Ankudinov and J. J. Rehr, Phys. Rev. B, 56, R1712 (1997); S. I. Zabinsky, J. J. Rehr, A. Ankudinov, R. C. Albers, and M. J. Eller, Phys. Rev. B, 52, 2995 (1995); for further details see, for example, the documentation at the URL: http://leonardo.phys.washington. edu/feff/. 